\title{
Stochastic Limit and Interacting Commutation Relations *
}

\author{
L. Accardi , I.Ya. Aref'eva ${ }^{\dagger}$, I.V.Volovich ${ }^{\ddagger}$ \\ Centro Vito Volterra \\ Università di Roma Torvergata
}

December 22, 2014

\begin{abstract}
It is shown in our previous work that in the stochastic limit of translation invariant Hamiltonians in quantum field theory the master field satisfies a new type of commutation relations, the so called entangled (or interacting) commutation relations. These relations extend the interacting Fock relations established earlier in non-relativistic QED and the free (or Boltzmann) commutation relations which have been found in the large $\mathrm{N}$ limit of QCD. In this paper a generalization of these results to the case of multiparticle external states is obtained.
\end{abstract}

* Dedicated to L.Streit

†Steklov Mathematical Institute, Gubkin St.8, GSP-1, 117966, Moscow, Russia, arefeva@mi.ras.ru

${ }^{\ddagger}$ Steklov Mathematical Institute, Gubkin St.8, GSP-1, 117966, Moscow, Russia, volovich@mi.ras.ru 


\section{Introduction}

In recent years there has been an interest in various modifications and deformations of the algebra of canonical commutation relations. Deformations are related with noncommutative geometry. Noncommutative geometry appears in physics in works of the founders of quantum mechanics. Heisenberg and Dirac have proposed that the phase space of quantum mechanics must be noncommutative and it should be described by quantum algebra. After works of Feynman it became clear that noncommutativity of phase space can be replaced by randomness of trajectories in the phase space.

New deformation schemes of canonical commutation relations presumably will use a new type of randomness. In the search of this we hope that new look at functional integration could be usefull $[1,2]$

There are two different view points on origins of deformations. In the first one deformations of canonical commutation relation appear via dynamics. In some special situations a deformation appears only in the spesial regime. Two examples are known $-1 / N$ expansion $[3,4]$ and stochastic limit [5]. The existence of new modified commutation relation could help to solve dynamical problems in the special regimes.

The second point of view on the deformation is different. It deals with at hoc modification of initial commutation relations $[6,7]$. This idea is closely related with noncommutative geometry [8,9] Motivated by investigations of quantum group symmetries in two dimensional integrable models [10] a proposal of consideration of noncommutative gauge symmetry on ordinary manifolds ("quantum group gauge theory") has been suggested in [11], see also $[12,13]$ for further discussions. Other approaches to field theory on noncommutative spaces and harmonic analysis have been discussed in $[14,16]$.

Especially simple relations

$$
b^{+} b=1
$$

appeared in the context of large $\mathrm{N}$ limit of matrix theories [3, 4]. in partucular, it is shown that a noncommutative gauge theory based on quantum Boltzmann relations (1.1) describes the large $\mathrm{N}$ limit in QCD. An attemps to find a phase integral description of systems quantized via (1.1) has been made in ([17]). An extension of the algebra (1.1) has been given in [18] as the algebra describing the interacting Fock space [19] obtained in the stochastic limit for non-relativistic QED.

In this paper we will consider the first approach to deformation of CCR and will consider a regime where a special deformation comes out.

In the previous paper we have prove [20] that the stochastic limit of interacting fields, under the only constraint of momentum conservation leads to a generalization of the algebra (1.1). We have obtained that the new algebra has as its generators the master field $B(p, k)$ depending on two momenta $p$ and $k$ and the operator density of particles $n(k)$ which satisfy the relations

$$
\begin{gathered}
B(p, k) B^{+}\left(p^{\prime}, k^{\prime}\right)= \\
\left.\left[n\left(p^{\prime}\right), B(p, k)\right]=\left(\delta\left(p^{\prime}-p\right)\right)-\delta\left(p^{\prime}-p+k\right)\right) B(p, k),
\end{gathered}
$$




$$
\left[n(p), n\left(p^{\prime}\right)\right]=0
$$

Here $E(p, k)$ is the energy associated to the interaction vertex.

We call the relations (1.2)-(1.4) entangled (or interacting) commutation relations because, on one hand they allow to calculate correlations of any order among the field and, on the other hand they show that the master fields are not kinematically independent. and, on the other hand they show that the master fields are not kinematically independent.

In the construction of these operators we use the Van Hove time rescaling $t \rightarrow t / \lambda^{2}$, $\lambda \rightarrow 0$ where $\lambda$ is the coupling constant. ${ }^{1}$. One can get a generalization of the algebra (1.2)-(1.4) for the multiparticle master field $B\left(p \mid k_{1}, \ldots, k_{n}\right)$.

Algebra (1.2)-(1.3) was found in [20] on one-particle subspace. In [20] was mentioned that in the stochastic limit on $n$-particle states survyve the diagrams that lead to (1.2)-(1.4). In this paper we perform this considerations explicitly. Namely, we will prove that only disconnected diagrams survive that connected parts consists on oneto-one half-planar components. Then we get an algebraic interpretation of surveving diagrams.

We find that instead of algebra of one master field $B(p, k)$ one has to introduce $n$ copies of master fields $B_{i}(p, k)$ its own field for each connected component.

The paper is organized as follows. In Sect. 2 there arae preliminary notations. In Sect. 3 we remind the definition of the stochastic limit. Sect. 4 contains the proof of the theorem about matrix elements of composite operators and in Sect. 5 we get an algebraic interpretation of the theorem.

\section{Notations and preliminaries}

\section{$2.1 \quad$ Space}

We use the following notations. The boson Fock space $\mathcal{F}$

$$
\begin{gathered}
\mathcal{F}=\bigoplus_{n=0}^{\infty} \mathcal{F}_{n}, \quad \mathcal{F}_{0}=C, \quad \mathcal{F}_{1}=L^{2}\left(R^{d}\right) \\
\mathcal{F}_{n}=\left(\mathcal{F}_{1}^{\otimes_{s}}\right)^{n}
\end{gathered}
$$

where the symbol $\otimes_{s}$ means the symmetric tenzor product. Elements $\phi \in \mathcal{F}$ are sets of symmetric functions

$$
\left\{\phi_{0}, \quad \phi_{1}, \ldots \phi_{n}, \ldots\right\}, \quad \phi_{n} \in L^{2}\left(R^{d n}\right)
$$

such that

$$
(\phi, \phi)=\left|\phi_{0}\right|^{2}+\sum\left(\phi_{n}, \phi_{n}\right)<\infty
$$

\footnotetext{
${ }^{1}$ To avoid a discussion of renormalization procedure we assume that there is an ultra-violet cut-off
} 
Let

$$
\mathcal{S}_{n}=\mathcal{S}\left(R^{d n}\right) \bigcup \mathcal{F}_{n}
$$

is the Schwarz space of well decrising at infinity symmetric functions. Denote

$$
\mathcal{S}=\bigoplus_{n}^{\infty}{ }^{\prime} \mathcal{S}_{n} \subset \mathcal{F}^{0}=\bigoplus_{n}^{\infty}{ }^{\prime} \mathcal{F}_{n} \subset \mathcal{F}
$$

where $\bigoplus_{n}^{\prime}$ means that we sum up only on finite sets, i.e. vectors from $\mathcal{S}$ and $\mathcal{F}^{0}$ have only finite number of non-zero components. The strong dual $\mathcal{S}^{\prime}$

$$
\mathcal{F} \subset \mathcal{S}^{\prime}=\prod \mathcal{S}_{n}^{\prime}
$$

is a direct product of space of symmetric tempered distributions. Creation and annihilation operators define the following linear maps $\mathcal{S} \rightarrow \mathcal{S}^{\prime}$

$$
\begin{gathered}
\left(a(k) \phi_{n}\right)\left(k_{1}, \ldots k_{n-1}\right)=\sqrt{n} \phi_{n}\left(k_{1}, \ldots k_{n-1}, k_{n}\right) \\
\left(a^{*}(k) \phi_{n}\right)\left(k_{1}, \ldots k_{n+1}\right)=\frac{1}{\sqrt{n+1}} \sum_{j}^{n+1} \delta\left(k-k_{j}\right) \phi_{n}\left(k_{1}, \ldots, \hat{k}_{j}, \ldots k_{n+1}\right)
\end{gathered}
$$

here the symbol $\hat{k}_{j}$ means that $k_{j}$ is omitted.

In the case of several type of particles

$$
\begin{gathered}
\mathcal{F}=\otimes_{i}^{(i)} \mathcal{F}, \quad{ }^{(i)} \mathcal{F}=\bigoplus_{n=0}^{\infty}{ }^{(i)} \mathcal{F}_{n},{ }^{(i)} \mathcal{F}_{0}=C,{ }^{(i)} \mathcal{F}_{1}=L^{2}\left(R^{d}\right) \\
{ }^{(i)} \mathcal{F}_{n}=\left({ }^{(i)} \mathcal{F}_{1}^{\otimes_{s}}\right)^{n}
\end{gathered}
$$

Creation and annihilation operators $a_{i}^{*}(k), a_{i}(k)$ satisfy the commutation relations

$$
\left[a_{i}(k), a_{j}^{*}\left(k^{\prime}\right)\right]=\delta_{i j} \delta\left(k-k^{\prime}\right)
$$

Let $v \in \mathcal{S}^{\prime}\left(R^{d(I+J)}\right)$. In this case the Wick monomial

$$
V_{I, J}=\int v\left(p_{1}, i_{1} \ldots p_{I}, i_{I} \mid q_{1}, j_{1} \ldots q_{J}, j_{J}\right) \prod_{l=1}^{I} a_{i_{l}}^{*}\left(p_{l}\right) d p_{l} \prod_{r=1}^{J} a_{j_{r}}\left(q_{r}\right) d q_{r}
$$

is a linear operator $V: \mathcal{S} \rightarrow \mathcal{S}^{\prime}$.

We will use for $V_{I, J}$ the Friedrichs graph representation. Each graph consists of one vertex and $I$ lines going from the vertex on the left and $J$ lines going on the right. The fist $I$ lines represent creation operators and the last $J$ lines represent annihilation. In what follows we will use the Wick theorem which represents a product of two Wick monomials as a sum of normal monomials:

$$
V_{I, J} W_{N, M}=: V_{I, J} W_{N, M}:+\sum_{t}^{\min \{J, N\}} V_{I, J} \underbrace{-\circ-}_{t} W_{N, M} .
$$


The numerical kernal of the Wick monomial : $V_{I, J} W_{N, M}:$ is

$$
v_{I, J} \otimes w_{N, M}
$$

$V_{I, J} \underbrace{-\circ-}_{t} W_{N, M}$ is a Wick monomial

$$
V_{I, J} \underbrace{-\circ-}_{t} W_{N, M}=\int\left(\prod_{r=1}^{I+N-t} d p_{r} a^{*}\left(p_{r}\right)\right) \cdot\left(\prod_{l=1}^{J+M-t} d q_{l} a\left(q_{l}\right)\right)(\underbrace{-\circ-w}_{t})\left(p_{1}, \ldots ; q_{1} \ldots\right)
$$

with the following numerical kernal

$$
\begin{gathered}
(v \underbrace{-\circ-w}_{t})\left(p_{1}, \ldots ; q_{1} \ldots\right)=C_{J}^{t} C_{N}^{t} t ! \int \prod_{r=1}^{t} d k_{r} \\
v_{I, J}\left(p_{1}, \ldots p_{I} ; k_{1}, \ldots k_{t}, q_{1}, \ldots q_{J-t}\right) w_{N, M}\left(k_{1}, \ldots k_{t} ; p_{I+1}, \ldots p_{I+N-1}, q_{J-t+1}, \ldots q_{J-t+M}\right)
\end{gathered}
$$

The equality (2.15) has a graphic representation as shown on (4).

$$
\text { file=acavst } 1 . e p s, \text { width }=450 \mathrm{pt}, \text { angle }=0
$$

Figure 1: Friedrich diagrams: graph representation of (2.15)

We will usein the main text also the following notations. The line of the grath is called the internal if it connects two vertecies of the graph. A graph is connected graph if all its vertecies are connected by a set of internal lines otherwise it is called a disconnected one. A connected graph is called one-particle redusible (1PI) if removing one line it becomes a disconected.

We also consider fermions. The standard notations are the following. The fermion Fock space $\hat{\mathcal{F}}$

$$
\begin{gathered}
\hat{\mathcal{F}}=\bigoplus_{n=0}^{\infty} \mathcal{F}_{n}, \quad \hat{\mathcal{F}}_{0}=C, \quad \hat{\mathcal{F}}_{1}=L^{2}\left(R^{d}\right) \\
\hat{\mathcal{F}}_{n}=\left(\hat{\mathcal{F}}_{1}^{\otimes_{a}}\right)^{n}
\end{gathered}
$$

where the symbol $\otimes_{a}$ means the asymmetric tenzor product. Elements $\psi \in \hat{\mathcal{F}}$ are sets of antisymmetric functions

$$
\left\{\psi_{0}, \quad \psi_{1}, \ldots \psi_{n}, \ldots\right\}, \quad \psi_{n} \in L^{2}\left(R^{d n}\right)
$$

such that

$$
(\psi, \psi)=\left|\psi_{0}\right|^{2}+\sum\left(\psi_{n}, \psi_{n}\right)<\infty
$$

Let

$$
\hat{\mathcal{S}}_{n}=\mathcal{S}\left(R^{d n}\right) \bigcup \hat{\mathcal{F}}_{n}
$$


is the Schwarz space of well decrising at infinity symmetric functions. Denote

$$
\hat{\mathcal{S}}=\bigoplus_{n}^{\infty} \hat{\mathcal{S}}_{n} \subset \hat{\mathcal{F}}^{0}=\bigoplus_{n}^{\infty} \hat{\mathcal{F}}_{n} \subset \hat{\mathcal{F}}
$$

where $\bigoplus_{n}^{\prime}$ means that we sum up only on finite sets, i.e. vectors from $\mathcal{S}$ and $\mathcal{F}^{0}$ have only finite number of non-zero components. The strong dual $\hat{\mathcal{S}}^{\prime} \hat{\mathcal{F}} \subset \hat{\mathcal{S}}^{\prime}=\prod \hat{\mathcal{S}}_{n}$ ' is a direct product of space of antisymmetric tempered distributions. Creation and annigilation fermionic operators are defined as a linear maps $\mathcal{S} \rightarrow \mathcal{S}^{\prime}$

$$
\begin{gathered}
\left(b(k) \psi_{n}\right)\left(k_{1}, \ldots k_{n-1}\right)=\sqrt{n} \psi_{n}\left(k_{1}, \ldots k_{n-1}, k_{n}\right) \\
\left(b^{*}(k) \psi_{n}\right)\left(k_{1}, \ldots k_{n+1}\right)=\frac{1}{\sqrt{n+1}} \sum_{j}^{n+1}(-1)^{j-1} \delta\left(k-k_{j}\right) \psi_{n}\left(k_{1}, \ldots, \hat{k}_{j}, \ldots k_{n+1}\right)
\end{gathered}
$$

here the symbol $\hat{k}_{j}$ means that $k_{j}$ is omitted.

In the case of several type of fermionis particles

$$
\begin{aligned}
\hat{\mathcal{F}}=\otimes_{i}{ }^{(i)} \hat{\mathcal{F}}, \quad{ }^{(i)} \hat{\mathcal{F}}= & \bigoplus_{n=0}^{\infty}{ }^{(i)} \hat{\mathcal{F}}_{n}, \quad{ }^{(i)} \hat{\mathcal{F}}_{0}=C, \quad{ }^{(i)} \hat{\mathcal{F}}_{1}=L^{2}\left(R^{d}\right) \\
& { }^{(i)} \hat{\mathcal{F}}_{n}=\left({ }^{(i)} \hat{\mathcal{F}}_{1}^{\otimes_{a}}\right)^{n}
\end{aligned}
$$

Creation and annihilation operators $b_{i}^{*}(k), b_{i}(k)$ satisfy the anticommutation relations

$$
\left\{b_{i}(k), b_{j}^{*}\left(k^{\prime}\right)\right\}=\delta_{i j} \delta\left(k-k^{\prime}\right)
$$

Let $v \in L_{2}\left(R^{d(I+J)}\right)$. In this case the Wick monom

$$
V_{I, J}=\int v\left(p_{1}, i_{1} \ldots p_{I}, i_{I} \mid q_{1}, j_{1} \ldots q_{J}, j_{J}\right) \prod_{l=1}^{I} b_{i_{l}}^{*}\left(p_{l}\right) d p_{l} \prod_{r=1}^{J} b_{j_{r}}\left(q_{r}\right) d q_{r}
$$

is a bounded linear operator $V: \hat{\mathcal{F}} \rightarrow \hat{\mathcal{F}}$.

\subsection{Hamiltonian}

We consider Hamiltonians of the form (??) where $H_{0}$ is a free Hamiltonian

$$
H_{0}=\sum_{i} \int \omega_{i}(k) a_{i}^{*}(k) a_{i}(k) d^{n} k
$$

and $V$ is a sum of Wick monoms. Operator (2.28) is self-adjoin in $\mathcal{F}$.

We consider two different types of Wick polinomials. The first type describes an interaction in the case when there is no translation invariance

$$
V=\sum_{I, J \geq 0} \int v\left(p_{1}, i_{1} \ldots p_{I}, i_{I} \mid q_{1}, j_{1} \ldots q_{J}, j_{J}\right) \prod_{l=1}^{I} a_{i_{l}}^{*}\left(p_{l}\right) d p_{l} \prod_{r=1}^{J} a_{j_{r}}\left(q_{r}\right) d q_{r}
$$


were $v\left(p_{1} \ldots p_{I} \mid q_{1} \ldots q_{J}\right)$ are some smooth functions. Hermition condition is

$$
v\left(p_{1} \ldots p_{I} \mid q_{1} \ldots q_{J}\right)=\bar{v}\left(q_{1} \ldots q_{J} \mid p_{1} \ldots p_{I}\right)
$$

In particular one can assume that

$$
v\left(p_{1} \ldots p_{I} \mid q_{1} \ldots q_{J}\right) \in \mathcal{S}\left(R^{I+J}\right) .
$$

Let $\mathcal{F}^{0}(\mathcal{S})=\bigoplus_{n}{ }^{\prime} \mathcal{S}\left(R^{d n}\right) \cap \mathcal{F}_{n}$ Under assumtion (2.31) we have

$$
V: \mathcal{S}^{0}(\mathcal{H}) \rightarrow \mathcal{F}^{0}(\mathcal{S})
$$

The second type is described by the translation invariant Hamiltonian

$$
\begin{gathered}
V=\sum_{I, J} V_{I, J}=\sum_{I, J} \int \hat{v}\left(p_{1}, i_{1}, \ldots p_{I}, i_{I} \mid q_{1}, j_{1} \ldots q_{J-1}, j_{J-1}, J_{j}\right) \\
\delta\left(\sum_{i}^{I} p_{l}-\sum_{j}^{J} q_{r}\right) \prod_{i=1}^{I} a_{i_{l}}^{*}\left(p_{l}\right) d p_{i} \prod_{j=1}^{J} a_{j_{r}}\left(q_{r}\right) d q_{r}
\end{gathered}
$$

Cleary the delta function causes the truble and there are singular terms in (2.32). Namely, $V_{I, 0} \phi_{n} n o \in \mathcal{F}$ unless $\phi_{n}=0$. This singularity is called a volume singularity. To give a meaning to hamiltonian with interaction (2.32) one has to introduce a volume cut-off then perform vacuum renormalization and vacuum dressing and only when remove cut-off. This procedure defines the hamiltonian in a new space (see [?] for details). To avoid this difficulty we will assume that for translation invariant interaction there are no pure creation and annigilation terms. In higher dimensions there are other singularities caused by the slow decrease of the kernel $\hat{v}$ at infinity. These singularities gives rise to the well known ultraviolet divergencies in quamtum field theory. In this paper we assume also that there is ultraviolet cutoff.

For fermions the interaction has the form (2.29) with $a^{\#} \rightarrow b^{\#}$ and the hermitian condition means now

$$
v\left(p_{1} \ldots p_{I} \mid q_{1} \ldots q_{J}\right)=\bar{v}\left(q_{J} \ldots q_{1} \mid p_{I} \ldots p_{1}\right)
$$

If one assumes that

$$
v\left(p_{1} \ldots p_{I} \mid q_{1} \ldots q_{J}\right) \in \mathcal{S}\left(R^{d(I+J)}\right)
$$

then operator $V$ is a bounded operator with $V^{*}=V$ and the full hamiltonian $H$ is selfadjoind on $D\left(H_{0}\right)$ and

$$
H \geq-|\lambda||| V||
$$

\section{The Stochastic Limit}

The stochastic limit is now widely used in the consideration of the long time/weak coupling behaviour of quantum dynamical systems with dissipation, see for example $[5]$. 
Let be given a quantum system described by the Hamiltonian

$$
H=H_{0}+\lambda V
$$

where $\lambda$ is the coupling constant. The starting point of the stochastic limit is the equation for the evolution operator in interaction picture

$$
\frac{d U^{(\lambda)}(t)}{d t}=-i \lambda V(t) U^{(\lambda)}(t)
$$

where

$$
V(t)=e^{i t H_{0}} V e^{-i t H_{0}}
$$

The main idea is that there exist a new quantum field (master field) and a new evolution operator $\mathcal{U}(t)$ (they both live on a space different from the original one) which approximates the old one

$$
U^{(\lambda)}(t) \approx \mathcal{U}\left(\lambda^{2} t\right)
$$

and the approximation is meant in the sense of appropriately chosen matrix elements. The above approximation suggests a natural interpretation of the van Hove rescaling [?] $\lambda \rightarrow 0, t \rightarrow \infty$ so that $\lambda^{2} t \sim$ constant $=\tau$ (new time scale): it means that we measure time in units of $1 / \lambda^{2}$ where $\lambda$ measures the strength of the self-interaction. By putting $\tau=1$ we see that the van Hove rescaling is equivalent to the time rescaling $t \rightarrow t / \lambda^{2}$, and therefore the limit $\lambda \rightarrow 0$ will capture the dominating contributions to the dynamics in the new time scale (the error can be estimated to be of order $\lambda^{2}$ ). It is remarkable that in this limit the dominating contributions can be explicitly resummed giving rise to a new unitary operator.

A simple change of variables shows that the time rescaling $t \rightarrow t / \lambda^{2}$ is equivalent to the following rescaling of the Schrödinger equation for the evolution operator:

$$
\frac{d U^{(\lambda)}\left(t / \lambda^{2}\right)}{d t}=-i \frac{1}{\lambda} V\left(t / \lambda^{2}\right) U^{(\lambda)}\left(t / \lambda^{2}\right)
$$

The unitary operator $\mathcal{U}(t)$ is then obtained by taking the limit $\lambda \rightarrow 0$ :

$$
\mathcal{U}(t)=\lim _{\lambda \rightarrow 0} U^{(\lambda)}\left(t / \lambda^{2}\right)
$$

and the corresponding limit equation is

$$
\frac{d \mathcal{U}(t)}{d t}=-i \mathcal{V}(t) \mathcal{U}(t)
$$

where

$$
\mathcal{V}(t)=\lim _{\lambda \rightarrow 0} \frac{1}{\lambda} V\left(\frac{t}{\lambda^{2}}\right)
$$

For a number important models the interaction Hamiltonian has the form

$$
V=\mathcal{A}+\mathcal{A}^{+}
$$


where $\mathcal{A}$ is a monomial in the creation and annihilation operators. The master field is given by the asymptotic behaviour of the collective operator

$$
\mathcal{A}_{\lambda}(t)=\frac{1}{\lambda} \mathcal{A}\left(\frac{t}{\lambda^{2}}\right)
$$

and its Hermitian conjugate. Here

$$
\mathcal{A}(t)=e^{i t H_{0}} \mathcal{A} e^{-i t H_{0}}
$$

The stochastic limit is meant in the sense of the convergence of correlation functions $<\mathcal{A}^{\epsilon_{1}}\left(t_{1} / \lambda\right) \ldots \mathcal{A}^{\epsilon_{n}}\left(t_{n} / \lambda\right)>$. Here $\epsilon_{i}= \pm$.

\section{Theorem about matrix elements of composite op- erators}

Let us consider the Hamiltonian

$$
H_{\lambda}=H_{0}+\lambda V
$$

where the free Hamiltonian

$$
\begin{gathered}
H_{0}=\int \varepsilon(p) c^{+}(p) c(p) d^{3} p+\int \omega(k) a^{+}(k) a(k) d^{3} k, \\
\left\{c(p), c^{+}\left(p^{\prime}\right)\right\}=\delta\left(p-p^{\prime}\right),\left[a(k), a^{+}\left(k^{\prime}\right)\right]=\delta\left(k-k^{\prime}\right)
\end{gathered}
$$

and the interaction Hamiltonian:

$$
V=\int d^{3} k d^{3} p g(k, p)\left(c^{+}(p) c(p-k) a(k)+h . c .\right)
$$

Here $g(k, p)$ is a test function and $\varepsilon(p)$ and $\omega(k)$ are one-particle dispersion laws, for example $\varepsilon(p)=p^{2} / 2, \omega(k)=|k|$.

The rescaled collective fields in this case have the form

$$
\begin{aligned}
& \mathcal{A}_{\lambda}(p, k, t)=\frac{1}{\lambda} e^{\frac{i t H_{0}}{\lambda^{2}}} c^{+}(p) a(k) c(p-k) e^{-\frac{i t H_{0}}{\lambda^{2}}}=\frac{1}{\lambda} c^{+}(p) a(k) c(p-k) e^{i t E(p, k) / \lambda^{2}} \\
& \mathcal{A}_{\lambda}^{+}(p, k, t)=\frac{1}{\lambda} e^{\frac{i t H_{0}}{\lambda^{2}}} c^{+}(p-k) a^{+}(k) c(p) e^{-\frac{i t H_{0}}{\lambda^{2}}}=\frac{1}{\lambda} c^{+}(p-k) a^{+}(k) c(p) e^{-i t E(p, k) / \lambda^{2}}
\end{aligned}
$$

where

$$
E(p, k)=\epsilon(p)-\omega(k)-\epsilon(p-k)
$$

is the corresponding energy. 
Let us consider the matrix $\left(n, n^{+}\right)$elements

$$
\left\langle 0\left|\prod_{j=1}^{n^{-}} c\left(q_{j}\right) \prod_{i=1}^{v} \mathcal{A}_{\lambda}^{\epsilon_{i}}\left(p_{i}, k_{i}, t_{i}\right) \prod_{j=1}^{n^{+}} c^{+}\left(q_{j}^{\prime}\right)\right| 0\right\rangle
$$

To evaluate (4.42) we apply Wick's theorem $n$ connected component contains. Each vertex contains 3 lines. The lines attached to vertex $i$ are characterized by two momenta $\left(k_{i}, p_{i}\right)$. We find the momentum corresponding to the 3rd line using the momentum conservation. Let a diagram consist on $N$ connected parts. Denote numbers of external lines coming in (coming out) $n$-connected part as $A_{n}\left(B_{n}\right)$. We have $\sum_{n} A_{n}=n^{-}$, $\sum_{n} B_{n}=n^{+}$. Let $L_{n}$ is a number of independent loops in $n$-connected part. We call the diagram corresponding to (4.42) as diagram with $\left(n^{-}, n^{+}\right)$external lines contains $L$ loops then only $L_{n}+A_{n}+B_{n}-1$ independent momenta ( $L_{n}$ momenta for loop variables and $A_{n}+B_{n}-1$ momenta for the exterior lines).

To formulate the main theorem let us remaind the definition of half-planar diagrams.

Definition 1. A diagram is calaled half-planar if can been drawn on a plane without self-intersection of the lines in such way that one can select a basic linea (in our case $c$-line) that all other lines are in upper half plane in the respect of the basic line.

TheOrem 1. The stochastic limit

$$
\lim _{\lambda \rightarrow 0}\left\langle 0\left|\prod_{j=1}^{n-} C\left(q_{j}\right) \prod_{i=1}^{v} \mathcal{A}_{\lambda}^{\varepsilon_{i}}\left(p_{i}, k_{i}, t_{i}\right) \prod_{j=1}^{n+} C^{+}\left(q_{j}^{\prime}\right)\right| 0\right\rangle
$$

exist as distributions. In this limit survive only diagrams with $n^{-}=n^{+}$connected parts. Each of these connected components disconnected in a sum of half-planar diagrams.

For every vertex there is the corresponding energy exponent. These energies depend on the momenta of the lines that enter to the given vertex,

$$
E_{i}^{ \pm}=E_{i}^{ \pm}\left(k_{i}, p_{i}\right)
$$

via dispersion laws,

$$
E^{ \pm}=[\epsilon(p)-\epsilon(p \pm k) \pm \omega(k)]
$$

In the proof of the theorem we will use the following lemma.

LEMMA 1. One has the following relation in the sense of distributions

$$
\lim _{\lambda \rightarrow 0} \frac{1}{\lambda^{2}} e^{i t E(p, k) / \lambda^{2}}=2 \pi \delta(t) \delta(E(p, k))
$$

The proof of the lemma is standard, see [21]. 
Let us consider a diagram corresponding to the matrix element (4.42). The proof of the theorem consists of three parts. First we prove that only diagrams consisting of pairs of conjugated vertices don't vanish in the limit $\lambda \rightarrow 0$. Definition of these diagrams will be given below. Next we prove that such diagrams consist on connected parts each of those contains one coming in and one coming out lines and moreover these connected parts are in fact non-crossing or half-planar diagrams. And finally we show that diagrams with $N$ connected components being half-planar (non-crossing) diagrams are described $N$ copies of operataors satisfying by the entangled commutation relations.

Generally, the sets of momenta corresponding to different vertices are different. However, it may happens that the same set of momenta corresponds to two different vertices. More precisely, momenta which come in the first vertex come out from the second one and viceversa.

Definition 2. We say that two incident vertices of a given connected diagram are conjugated if the momenta coming in the first vertex come out from the second vertex, i.e. the vertices have the same momenta but with the opposite orientation.

If the $i$-vertex has a conjugated vertex then we denote the latter by $\hat{i}$. A typical example of diagrams containing at least one pair of conjugater vertices is a diagram with a mass insertion such that this insertion contains a line that does not cross others lines of the diagram (see Fig.2).

file $=$ acavst2.eps, width $=150 \mathrm{pt}$, angle $=0$

Figure 2: Diagram with a pair of conjugated vertices

Another simple example if a diagram containing only conjugated vertices is a disconnected diagram with two connected parts each of them is the second order mass insertion (Fig.3).

$$
\text { file=acavst3.eps, width }=100 p t \text {, angle }=0
$$

Figure 3: Disconnected diagram with two pairs of conjugated vertices

On fig. 4 we present a diagram with a pair of conjugated vertices.

Let us prove the main

$$
\text { file }=\text { acavst } 4 \text {.eps, width }=350 \text { pt, angle }=0
$$

Figure 4: Diagram with a pair of conjugated vertices 
LEMMA 2. If a connected diagram doesn't consist only from pairs of conjugated vertices then it vanishes in the limit $\lambda \rightarrow 0$ (in the sense of distributions).

Proof. To a given diagram, representing a matrix element (4.42) being integrated over $t_{1}, \ldots, t_{v}$ with test functions, corresponds the expression that schematically can be written as

$$
\frac{1}{\lambda^{v}} \int e^{i \sum_{i=1}^{v} E_{i} t_{i} / \lambda^{2}} \phi(t, p, q) \prod_{a}^{A+B-1} d p_{a} \prod_{l=1}^{L} d q_{l} \prod_{i=1}^{v} d t_{i}
$$

here by $t$ we mean $t_{1}, \ldots, t_{v}$, by $p$ we mean $p_{1}, \ldots, p_{A}, p_{1}^{\prime}, \ldots, p_{B}^{\prime}$ and $q$ denotes the set of independent momenta associated with the diagram under consideration. $E_{i}$ are given by (4.43) and $\phi(t, p, q)$ is a test function.

To evaluate the asymptotic behaviour of this expression when $\lambda \rightarrow 0$ we will make the change of variables corresponding to the conjugated vertices. Suppose that the number of vertices $v$ should be even, $v=2 n$. Suppose also that there are $n_{0}$ pairs of conjugated vertices which are denoted $\left\{i_{1}, \hat{i}_{1}, \ldots, i_{n_{0}}, \hat{i}_{n_{0}}\right\}$. Let us divide the set of all vertices $\{1, \ldots, 2 n\}$ into two disjoint subsets $\left\{i_{1}, i_{2}, \ldots, i_{n_{0}}, i_{n_{0}+1}, \ldots, i_{n}\right\}$ and $\left\{\hat{i}_{1}, \hat{i}_{2}, \ldots, \hat{i}_{n_{0}}, i_{n+n_{0}+1}, \ldots i_{2 n}\right\}$ such that in every subset there are no conjugated vertices. We denote the corresponding set of time variables $\left\{t_{i_{1}}, \ldots t_{i_{n}}\right\}$ by $t^{(1)}$ and the set $\left\{t_{\hat{i}_{1}}, \ldots t_{\hat{i}_{n_{0}}}, t_{i_{n+n_{0}+1}}, \ldots t_{i_{2 n}}\right\}$ by $t^{(2)}$.

Now we perform the following change of variables

$$
\begin{gathered}
\left(t^{(1)}, t^{(2)}\right) \rightarrow\left(\tau, t^{(2)}\right) \\
t^{(1)}=t^{(2)}+\lambda^{2} \tau,
\end{gathered}
$$

or more precisely

$$
\begin{gathered}
\left(t^{(1)}, t^{(2)}\right)=\left(t_{1}, \ldots, t_{2 n}\right) \rightarrow\left(\tau, t^{(2)}\right)=\left(\tau_{1}, \ldots, \tau_{n} ; t_{\hat{i}_{j}}, j=1 \ldots, n_{0} ; t_{i_{n+r}}, r=n_{0}+1, \ldots, n\right) \\
t_{i_{j}}=t_{\hat{i}_{j}}+\lambda^{2} \tau_{j}, \quad 1 \leq j \leq n_{0} \\
t_{i_{j}}=t_{i_{n+j}}+\lambda^{2} \tau_{j}, \quad n_{0}<j \leq n
\end{gathered}
$$

After this the integral (4.44) takes the following form

$$
\begin{gathered}
\int e^{i \sum_{j=1}^{n} \tau_{j} E_{i_{j}}} e^{i \sum_{j=1}^{n_{0}}\left(E_{i_{j}}+E_{\hat{i}_{j}}\right) t_{\hat{i}_{j}} / \lambda^{2}} \cdot e^{i \sum_{j=n_{0}+1}^{n}\left(E_{i_{j}}+E_{i_{n+j}}\right) t_{i_{n+j}} / \lambda^{2}} \\
\phi\left(t^{(2)}+\lambda^{2} \tau, t^{(2)}, p, q\right) \cdot \prod_{j=1}^{n} d \tau_{j} \prod_{j=1}^{n_{0}} d t_{\hat{i}_{j}} \prod_{j=n_{0}+1}^{n} d t_{i_{n+j}} \prod d p \cdot \prod d q
\end{gathered}
$$

By definition of conjugated vertices $E_{i_{j}}+E_{\hat{i}_{j}}=0$ and we left with

$$
\begin{gathered}
\int e^{i \sum_{j=1}^{n} \tau_{j} E_{i_{j}}} e^{i \sum_{j=n_{0}+1}^{n}\left(E_{i_{j}}+E_{i_{n+j}}\right) t_{i_{n+j}} / \lambda^{2}} \\
\phi\left(t^{(2)}+\lambda^{2} \tau, t^{(2)}, p, q\right) \cdot \prod_{j=1}^{n} d \tau_{j} \prod_{j=1}^{n_{0}} d t_{\hat{i}_{j}} \prod_{j=n_{0}+1}^{n} d t_{i_{n+j}} \prod d p \cdot \prod d q
\end{gathered}
$$


Here $t^{(2)}+\lambda^{2} \tau$ schematically represents the dependence of the half of the $t$-variables on $\lambda$. When $\lambda \rightarrow 0$ we can neglect the dependence of $\phi$ on $\lambda$ and the integration over $\tau$ gives the product of $\delta\left(E_{i_{j}}\right)$

$$
\int \prod_{j=1}^{n} \delta\left(E_{i_{j}}\right) e^{i \sum_{j=n_{0}+1}^{n}\left(E_{i_{j}}+E_{i_{n+j}}\right) t_{i_{n+j}} / \lambda^{2}} \phi(t, t, p, q) \prod_{j=n_{0}+1}^{n} d t_{i_{n+j}} \prod d p \prod d q
$$

Note that the second exponent in the expression (4.50) vanishes since the energies in the conjugated vertices are equal.

Suppose that in the diagram there are non-conjugated vertices, i.e. $n \neq n_{0}$. When $\lambda \rightarrow 0$ the expression (4.51) goes to zero since, according to our assumption the set of momenta in vertices $i_{j}$ and $i_{n+j}, n_{0}<j \leq n$ do not coincide and therefore the functions $E_{i_{j}}+E_{i_{n+j}}$ as functions of momenta don't vanish and therefore according the Riemann-Lebesgue lemma we get zero in the limit $\lambda \rightarrow 0$.

In the case when $n=n_{0}$ the exponent in (4.51) vanishes and generally we get the non-zero answer:

$$
\int\left(\prod_{i=1}^{n} \delta\left(E_{i_{j}}\right)\right) \phi\left(\left\{t_{i_{j}}\right\},\left\{t_{i_{j}}\right\}, p, q\right) \prod d p \prod d q
$$

Suppose now $v=2 n+1$. In this case the given diagram cannot be divided into pains by trivial raison and therefore its contribution goes to zero with $\lambda \rightarrow 0$. More explicitly. For the case of odd $v, v=2 n-1$, we once again select $n$ vertices and make the following change of variables

$$
\left(t_{1}, \ldots, t_{2 n-1}\right) \rightarrow\left(\tau_{1}, \ldots, \tau_{n} ; t_{\hat{i}_{j}}, j=1, \ldots, n_{0} ; t_{i_{n+r}}, r=1, \ldots, n-1-n_{0}\right)
$$

with the $\tau_{i}$ as before (see (4.47)).

$$
\begin{gathered}
t_{i_{j}}=t_{\hat{i}_{j}}+\lambda^{2} \tau_{j}, \quad 1 \leq j \leq n_{0} \\
t_{i_{j}}=t_{i_{n+j}}+\lambda^{2} \tau_{j} \quad n_{0}+1 \leq j \leq n-1 \\
t_{i_{n}}=\lambda^{2} \tau_{n}
\end{gathered}
$$

The difference with the case of even $v$ is that we get an extra factor $\lambda$ in front of the integral

$$
\begin{gathered}
\lambda \int e^{i \sum_{j=1}^{n} \tau_{j} E_{i_{j}}} e^{i \sum_{j=1}^{n_{0}}\left(E_{i_{j}}+E_{\hat{i}_{j}}\right) t_{\hat{i}_{j}} / \lambda^{2}} e^{i \sum_{j=n_{0}+1}^{n-1}\left(E_{i_{j}}+E_{i_{n+j}}\right) t_{i_{n+j}} / \lambda^{2}} \\
\phi(t+\lambda \tau, t, p, q) \prod d p d q \prod_{j=1}^{n} d t_{j} \prod_{j=n_{0}}^{n_{0}} d t_{i_{n+j}}
\end{gathered}
$$

Here we use the same schematical notations as in (??). When $\lambda \rightarrow 0$ we neglect the $\lambda$-dependence of $\phi$ and we get a product of $\delta$-functions. The second exponent goes 
out. The third exponent disappears in the case then $n_{0}=n-1$, but since we have an extra factor $\lambda$ the expression (4.54) always goes to zero as $\lambda \rightarrow 0$.

The lemma is proved.

LEMma 3. If a diagram with $\left(n^{-}, n^{+}\right)$external lines consists only from pairs of conjugated vertices then $n^{-}=n^{+}$and the diagram has on $n^{+}$connected parts. Each connected diagram has $(1,1)$ external lines and is half-planar, i.e. it can be drown in the half-plane without self intersections.

We will not present here the simple proof of this lemma.

Now the theorem follows from the above three lemma.

\section{$5 \quad$ Entangled Commutation Relations}

\subsection{Stochastic limit for $N$ point correlators between one-particle states}

The following theorem has been proved in [AcArVo].

THEOREM 2. The stochastic limit

$$
\lim _{\lambda \rightarrow 0} \mathcal{A}_{\lambda}(p, k, t)=B^{-}(p, k, t), \quad \lim _{\lambda \rightarrow 0} \mathcal{A}_{\lambda}^{+}(p, k, t)=B^{+}(p, k, t)
$$

exists in the sense of the convergence of the matrix elements $\left(\epsilon_{i}= \pm\right.$ )

$$
\begin{gathered}
\lim _{\lambda \rightarrow 0}<0\left|c(q) \mathcal{A}_{\lambda}^{\epsilon_{1}}\left(p_{1}, k_{1}, t_{1}\right) \ldots \mathcal{A}_{\lambda}^{\epsilon_{n}}\left(p_{n}, k_{n}, t_{n}\right) c^{+}\left(q^{\prime}\right)\right| 0>= \\
\left(\Psi_{0}, c(q) B^{\epsilon_{1}}\left(p_{1}, k_{1}, t_{1}\right) \ldots B^{\epsilon_{n}}\left(p_{n}, k_{n}, t_{n}\right) c^{+}\left(q^{\prime}\right) \Psi_{0}\right)
\end{gathered}
$$

as distributions and the limiting operators $B^{-}=B$ and $B^{+}$satisfy the entangled commutation relations

$$
\begin{gathered}
B(p, k, t) B^{+}\left(p^{\prime}, k^{\prime}, t^{\prime}\right)=2 \pi \delta\left(t-t^{\prime}\right) \delta\left(p-p^{\prime}\right) \delta\left(k-k^{\prime}\right) \cdot \delta(E(p, k)) n(p) \\
{\left[n\left(p^{\prime}\right), B^{\mp}(p, k, t)\right]=( \pm)\left(\delta\left(p^{\prime}-p\right)-\delta\left(p^{\prime}-p+k\right)\right) B^{\mp}(p, k, t)} \\
{\left[n(p), n\left(p^{\prime}\right)\right]=0}
\end{gathered}
$$

Here $\Psi_{0}$ is the vacuum in the new Hilbert space, $B(p, k, t) \prod_{i} c^{+}\left(q_{i}\right) \Psi_{0}=0$. We use the same notations for the creation and annihilation operators of c-particles in the original and in the new Hilbert spaces. $n(p)$ is the operator density of the c-particles, $n(p)=c^{+}(p) c(p)$. 
If we set

$$
B(p, k, t)=b(t) \otimes B(p, k)
$$

where

$$
b(t) b^{+}\left(t^{\prime}\right)=2 \pi \delta\left(t-t^{\prime}\right)
$$

then we get the relations (1.2)

$$
B(p, k) B^{+}\left(p^{\prime}, k^{\prime}\right)=n(p) \delta(E(p, k)) \delta\left(p-p^{\prime}\right) \delta\left(k-k^{\prime}\right)
$$

The more general theorem will be proved in the next section. Note that to get non-zero in the RHS of (5.56) we have to chose suitable dispersion relations, so that there are non-trivial solutions of equation $E(p, k)=0$.

\subsection{Stochastic limit for $N$ point correlators between $n$ parti- cles states}

In this section the main result of this paper will be proved.

Let us consider the matrix element

$$
\left\langle\Phi_{n}^{\prime}, \prod_{i=1}^{N} \mathcal{A}_{\lambda}^{\varepsilon_{i}} \Phi_{m}\right\rangle
$$

where $\mathcal{A}_{\lambda}$ and $\mathcal{A}_{\lambda}^{+}$are given by (4.39) and (4.40) and

$$
\begin{gathered}
\Phi_{m}=\prod_{j=1}^{m} c^{+}\left(f_{j}\right)\left|0>; \quad \Phi_{n}^{\prime}=\prod_{j=1}^{n} c^{+}\left(f_{j}^{\prime}\right)\right| 0>, \\
c^{+}(f)=\int c^{+}(k) f(k) d k, \quad c\left(f^{\prime}\right)=\int c(k) f^{\prime}(k) d k
\end{gathered}
$$

Lemma 2 claims that if diagram corresponding to (5.59) doesn't consist only of pairs of conjugated vertices then its contribution vanishes in the limit $\lambda \rightarrow 0$ (in the sense of distributions). One can see that to select diagrams corresponding to $(n 1)$ such that all their vertices form pairs of conjugated vertices one has to consider diagrams that satisfy the following requirements:

a) $n=m$

b) For $n \neq 1$ diagram must be disconnected and it must include $n$ connected parts.

Each connected part corresponds to $N_{i}$ point correlator of composite operators between one particle stats and

$$
\sum_{i=1}^{n} N_{i}=N
$$

c) Each connected part contains only pairs of conjugated vertices. 
To describe all diagrams satisfying above requirements it is suitable to do the following. Let us first apply the Wick theorem to represent the product $\mathcal{A}$ 's of in normal form in respect only of operators $c$ and $c^{+}$

$$
\prod_{i=1}^{N} \mathcal{A}^{\varepsilon_{i}}=\sum_{\rho}: K_{\rho,\left\{\varepsilon_{i}\right\}}\left(a, a^{+}, c, c^{+}\right):_{c}
$$

here : $:_{c}$ means the normal product in respect of operators $c$ and $c^{+}$, and summation on $\rho$ involves all terms that appear in the process of an application of the Wick theorem. Applying the Wick theorem to

$$
\prod_{i} c\left(f_{i}^{\prime}\right) K_{\rho} \prod_{j} c^{+}\left(f_{j}\right)
$$

We have

$$
\begin{gathered}
\prod_{i}^{n} c\left(f_{i}^{\prime}\right) \prod_{i=1}^{N} \mathcal{A}_{\lambda}^{\varepsilon_{i}} \prod_{j=1}^{n} c^{+}\left(f_{i}\right)= \\
=\sum_{l=0}^{n} \sum_{r=0}^{n} \sum_{\rho}:\left(\prod_{j=1}^{n} c\left(f_{j}^{\prime}\right)\right) \frac{c}{l} K_{\rho,\left\{\varepsilon_{i}\right\}} \frac{c}{r}\left(\prod_{i=1}^{n} c^{+}\left(f_{i}\right)\right):_{c}
\end{gathered}
$$

symbol $\frac{c}{l}$ means that we take into account the diagram with $l$ contraction ( $c$-lines).

For example,

$$
\begin{gathered}
\mathcal{A}_{\lambda}^{+} \mathcal{A}_{\lambda}=K_{1,+-}+K_{2,+-} \\
K_{1,+-}=\int d p d p^{\prime} d k d k^{\prime} a^{+}(k) c^{+}(p-k) c^{+}\left(p^{\prime}\right) c(p) c\left(p^{\prime}-k^{\prime}\right) a\left(k^{\prime}\right) v(p, k) \bar{v}\left(p^{\prime} k^{\prime}\right) \\
K_{2,+-}=\int d p d k d k^{\prime} c^{+}(p-k) a^{+}(k) a\left(k^{\prime}\right) c\left(p-k^{\prime}\right) v(p, k) \bar{v}(p, k)
\end{gathered}
$$

(see fig. 5)

$$
\text { file=acavst5.eps, width }=450 \mathrm{pt} \text {, angle }=0
$$

Figure 5: Friedrich diagrams representation of (5.62)

For the product of $\mathcal{A A}^{+}$we have

$$
\begin{gathered}
\mathcal{A}_{\lambda} \mathcal{A}_{\lambda}^{+}=K_{1,-+}+K_{2,-+} \\
K_{1,-+}=\int d p d p^{\prime} d k d k^{\prime} v(p, k) \bar{v}\left(p^{\prime}, k^{\prime}\right) c^{+}(p) c^{+}\left(p^{\prime}-k^{\prime}\right) c(p-k) c\left(p^{\prime}\right) a(k) a^{+}\left(k^{\prime}\right) \\
K_{2,-+}=\int d p d k d k^{\prime} v(p, k) \bar{v}\left(p-k+k^{\prime}, k^{\prime}\right) c^{+}(p) c\left(p-k+k^{\prime}\right) a(k) a\left(k^{\prime}\right)
\end{gathered}
$$




$$
\text { file=acavst6.eps, width }=450 \mathrm{pt}, \text { angle }=0
$$

Figure 6: Friedrich diagrams representation of (5.63)

(see fig. 5)

To select the non-zero vacuum avarage of (5.61) that consist of diagrams satisfying requirements a), b) and c) we take the factorized products

$$
\sum_{\sum j_{i}=N} \prod_{i=1}^{n}\left(c\left(f_{i}^{\prime}\right) \frac{c}{1} A_{\lambda}^{\varepsilon_{j_{1}}} \overline{1} \frac{c}{1} A_{\lambda}^{\varepsilon_{j_{2}}} \frac{c}{1} \ldots A_{\lambda}^{\varepsilon_{j_{k}}} \frac{c}{1} c\left(f_{l_{i}}\right)\right)
$$

Now (5.64) is a sum of products of Wick monomials containing $a$ and $a^{+}$operators.

To select only diagrams with pairs of conjugated vertices we perform the following. In $i$-component

$$
c\left(f_{i}^{\prime}\right) \frac{c}{1} A_{\lambda}^{\varepsilon_{j_{i}}} \frac{c}{1} \ldots A_{\lambda}^{\varepsilon_{j_{i}}} \frac{c}{1} c\left(f_{l_{i}}\right)
$$

we substitute instead of $a^{\varepsilon}$ operators the products of the Boltzmannian operators $b_{i}^{\varepsilon}$, white noise operators $b_{i}(t)$ and "square root" of energy $\delta$-function. $b_{i}^{\varepsilon}$ satisfy the relations

$$
b_{i}(k) b_{i^{\prime}}^{+}\left(k^{\prime}\right)=\delta_{i i^{\prime}} \delta\left(k-k^{\prime}\right)
$$

and white noise operators $b_{i}(t)$ satisfy the relations,

$$
b_{i}(t) b_{i^{\prime}}\left(t^{\prime}\right)=2 \pi \delta_{i i^{\prime}} \delta\left(t-t^{\prime}\right)
$$

Diagrams corresponding to (5.59) that survive under limit $\lambda \rightarrow 0$ consist on $n$ continuous $c$-lines connected one annihilator operator, some number of vertices and one creator operators. These c-lines enter in different connected parts of one disconnected diagram. Beside $c$-lines diagrams contain $a$-lines, that connecte only vertices that are crossed by the same $c$-line. Each of connected part is representing by a half-planar diagram.

Above consideration proves the following

Theorem 3. The stochastic limit of the matrix element $(n 1)$ can be represented as follows

$$
\begin{gathered}
\lim _{\lambda \rightarrow 0}\left\langle 0\left|\prod_{j=1}^{n} c\left(f_{j}^{\prime}\right) \prod_{i=1}^{N} \mathcal{A}_{\lambda}^{\varepsilon_{i}} \prod_{j=1}^{m} c^{+}\left(f_{j}\right)\right| 0\right\rangle= \\
=\delta_{n m} \sum_{p \in \mathcal{P}_{n}} \sum_{\text {"partitions" }}\left\langle 0\left|c\left(f_{1}^{\prime}\right) B^{\varepsilon_{i_{1}}} \ldots B^{\varepsilon_{i_{k_{1}}}} c^{+}\left(f_{p(1)}\right)\right| 0\right\rangle \cdot \\
\cdot\left\langle 0\left|c\left(f_{2}^{\prime}\right) B^{\varepsilon_{j_{1}}} \ldots \varepsilon^{j_{k_{2}}} c^{+}\left(f_{p(2)}\right)\right| 0\right\rangle \ldots\left\langle 0\left|c\left(f_{n}^{\prime}\right) B^{\varepsilon_{l_{1}}} \ldots B^{\varepsilon_{l_{k n}}} c^{+}\left(f_{p(n)}\right)\right| 0\right\rangle
\end{gathered}
$$

Here $\mathcal{P}_{n}$ is the group of transpositions and the sum over "partitions" includes the sum over all subsets $\left(i_{1}, \ldots, i_{k_{1}}\right)\left(j_{1}, \ldots, j_{k_{2}}\right) \ldots\left(l_{1}, \ldots, l_{k_{n}}\right)$ of $\{1, \ldots, N\}$ preserving the order $k_{1}+k_{2}+\ldots+k_{n}=N$. 


\section{Conclusion}

The theorem from the previous section describes the stochastic limit of composite operators states. It gives the second quantized generalization on multiparticle of interacting commutation relations from the previous works [5,20]. It would be interesting to find an operator expression of the obtained fomula as well as a corresponding path integral representation.

Acknowledgments. I.Ya.A. and I.V.V. are grateful to the Centro Vito Volterra Universita di Roma Tor Vergata for the kind hospitality. This work is supported in part by INTAS grant 96-0698, I.Ya.A. is supported also by RFFI-99-01-00166 and I.V.V. by RFFI-99-01-00105

\section{References}

[1] L. Streit, A new look at functional integration. In Capri 1993, Proceedings, Advances in dynamical systems and quantum physics* $307-325$.

[2] L. Streit, White noise analysis and functional integrals. UMA-MAT-11-93

[3] I.Ya.Aref'eva and I.V.Volovich. Large $N Q C D$ and q-deformed quantum field theories, Nucl.Phys.B 462 (1996) 600

[4] M. B. Halpern and C. Schwartz, The Algebras of Large N Matrix Mechanics, hepth/9809197.

[5] L. Accardi, Y.G. Lu and I.V.Volovich, Quantum theory and its stochastic limit, Springer Acad. Press, (in press)

[6] O. Greenberg, Phys.Rev.Lett. 64 (1990) 705.

[7] I.Ya. Aref'eva and I.V. Volovich, Quantum group particles and non-archimedean geometry, Phys. Lett. B268(1991)179-187

[8] A.Connes, Noncommutative Geometry, Academic Press, 1994.

[9] J.Madore, "An Introduction to Noncommutative Differential Geometry and its Physical Applications", Cambridge Univ. Press, 1995.

[10] L.D.Faddeev, N.Reshetishin and L.Taxhtajain. Algebra and Analysis, 1 (1987) 1.

[11] I.Ya.Aref'eva and I.V.Volovich. Quantum group gauge theory, Mod.Phys.Lett.A6(1991) 893-907.

[12] A.P.Isaev and Z.Popowicz, Phys.Lett. B307 (1993) 353-361

[13] L.Castellani, $U_{q}(N)$ Gauge Theories, Mod.Phys.Lett. A9 (1994) 2835-2848

[14] A. Jaffe, Quantum Harmonic Analysis and Geometric Invariants, Physics/9709011 
[15] I.Ya. Aref'eva and I.V. Volovich, Noncommutative Gauge Fields on Poisson Manifolds, hep-th/9907114

[16] J.Lukierski, Deformed Quantum Relativistic Phase Spaces - an Overview, hepth/9812063.

[17] I.Ya.Aref'eva and A.P.Zubarev, unpublished

[18] L. Accardi, Y.G. Lu and I.V. Volovich, Interacting Fock spaces and Hilbert module extensions of the Heisenberg commutation relations, Publications of IIAS, Kyoto, 1997

[19] L. Accardi, Y.G. Lu, Comm. Math. Phys. 180 (1996) 605

[20] L. Accardi, I.Ya. Aref'eva and I.V. Volovich Non-Equilibrium Quantum Field Theory and Entangled Commutation Relations, hep-th/9905035

[21] V.S.Vladimirov, 